\title{
Lifestyle differentiation among older adults: exploring the links between individuals' behaviours, socio-demographic characteristics, health and wellbeing in later life
}

\author{
Ewa Jarosz ${ }^{1,2}$ (D) \\ ${ }^{1}$ Institute of Philosophy and Sociology, Polish Academy of Sciences, Warsaw, Poland and ${ }^{2}$ SYNYO, Vienna, \\ Austria Email: ejarosz@ifispan.edu.pl
}

(Accepted 22 September 2021)

\begin{abstract}
The association between everyday activities, health and subjective wellbeing in older adults has mostly been examined using different activities as separate variables. Which activities are likely to come together in individuals' daily time-use patterns, or in what context, has not yet been analysed. This study looks at a broad range of spontaneously reported activities, their location and social context to identify latent behavioural classes. The data used in the study came from a sample of 200 non-institutionalised adults aged 65 and above. Activity data were collected using the Experience Sampling Method. Generalised structural equation modelling was used to identify the classes. Three distinctive behavioural classes, representing different lifestyles, emerged: passive domiciliary, active functional and social recreational. They constituted 30,53 and 17 per cent of the sample, respectively. Class membership was related to individuals' age, education and selected dimensions of health measured using the Nottingham Health Profile: energy levels and emotional response. There was consistency between the objectively measured class and an individual's subjective assessment of their physical and emotional health. While both class membership and subjective wellbeing were associated with health, the relationship between class and wellbeing was weak and fully explained by socio-demographic and health-related variables.
\end{abstract}

Keywords: lifestyle; behaviours; activities; ageing; subjective wellbeing; health

\section{Introduction}

The association between everyday activities, health and wellbeing in older adults has received substantial theoretical and research attention. Studies have found that a greater frequency of participation in certain types of activities is associated with better long-term outcomes including health, wellbeing and survival (Menec, 2003; Agahi and Parker, 2008; Adams et al., 2011). Overall, the range of activities 
which have been shown to influence wellbeing is very broad, but their effect on wellbeing may differ depending on the individual's socio-demographic characteristics (Luo et al., 2019).

The concept of lifestyle is rarely researched in this context. While initially lifestyle described conspicuous behavioural patterns, social conduct and consumption, with time the concept was expanded to include a broad range of everyday practices such leisure, eating or socialising (Sobel, 1981). In a lifestyle-based approach to individuals' behaviours, such as one adopted by the health lifestyle theory (Cockerham, 1999) or by structural constructivism (Bourdieu, 1984), behaviours or practices are seen in a synthesised manner, and they are linked with one another through their common denominators such as structural position or class habitus. Such a synthesised approach is uncommon in the field of gerontology, where studies looking at the time use of older adults typically focus on selected activities as independent units, not as a reflection of unobservable dispositions.

This study examined older adults' daily activities and their context as observable indicators of the different lifestyles adopted by older adults to match their preferences and abilities. It used information on a broad range of spontaneously reported daily activities as well as where and with whom they took place. There were three research objectives. First, to identify the different lifestyles of older adults - using a bottom-up approach that looked at the daily activities and their contextual characteristics as indicators. Second, to investigate how these lifestyles were associated with individuals' socio-demographic characteristics, self-rated general health and the detailed dimensions of health: energy levels, emotional response, social isolation and sleep disturbances. Third, it analysed the association between individuals' lifestyle, structural position, health and subjective wellbeing.

\section{Literature review}

\section{Theoretical views on the role of activities in later life}

Rewarding daily activities are seen as an essential component of living a 'good life' in old age. Their importance has been acknowledged in the activity theory of ageing (Havighurst, 1961; Lemon et al., 1972), the studies on the quality of life of older adults (Raphael et al., 1997), and reflected in the classic version of the concept of successful ageing which lists good physical functioning and 'active engagement with life' as key indicators of successful ageing (Rowe and Kahn, 1987, 1997). Noteworthy, the types of activities seen as contributing to good ageing are not fixed and vary depending on the theoretical approach. An alternative perspective on successful ageing offered by the psychosocial approach (Baltes and Baltes, 1990; Kahana and Kahana, 1996) frames it in terms of a positive adaptation to increasing limitations and challenges related to ageing. In this case, good ageing would translate to the ability to maintain social roles, compensate for losses and safeguard wellbeing in the face of increasing functional limitations. In behavioural terms, the psychosocial approach emphasises selecting appropriate leisure and meaningful social activities (Adams, 2004).

The concept of the Valuation of Life (Lawton et al., 1999) is another take on the foundations of a good life and mental resilience in late adulthood. It postulates that there is a strong link between activities and positive health and wellbeing outcomes, 
but does not emphasise particular activities (Moss et al., 2007). Studies on the Valuation of Life conclude that there is no one-size-fits-all approach. Different aspects of life may be endorsed by different older adults to maintain a positive valuation of life while adapting to changes associated with ageing (Jopp et al., 2008). Activity participation is seen as a way to fulfil personal goals and, regardless of the nature of a particular activity, add meaning to life and boost wellbeing (Lawton et al., 2002). Review studies on activities and wellbeing generally support this view and state that not all activities are equally appealing to all older adults (Adams et al., 2011). There are various ways of living which can promote wellbeing in late adulthood, and they depend on individuals' characteristics, their health and preferences.

\section{Differentiation and the significance of daily activities}

Everyday time-use patterns of older adults are socially differentiated. Differentiation runs along the lines of the basic socio-demographic characteristics, primarily individuals' age and gender (Gauthier and Smeeding, 2003), marital status (Chen et al., 2015), educational attainment (Zhu et al., 2017) and social status (Chen et al., 2012). Living arrangements and area of residence may also affect daily behaviours. Living with others, as opposed to living alone, may facilitate social activities, as it generates opportunities for meaningful social interaction (Ayalon, 2009). Area of residence affects daily lives, in particular for individuals with functional limitations. Older adults living in rural areas face greater obstacles to mobility and activity choices, which results in less travel and overall more monotonous lifestyles (Li, 2006).

Daily activities are also closely linked with an individual's health condition, which has been seen as both a determinant and an outcome. On the one hand, a high share of passive leisure may be indicative of living with a disability or having long-term health problems (Espinel et al., 2015). On the other, greater physical activity has been linked with better long-term physical health and wellbeing (Mullen et al., 2011), and predicted lower rates of cognitive decline among older adults (Yaffe et al., 2001). Overall, studies have analysed a broad range of different activities and found different associations with both positive or negative health outcomes. Involvement in productive activities, such as work, informal help, care work or volunteering, has been associated with better health and wellbeing (Luo et al., 2019). Socialising (Talmage et al., 2020), child care (Drew and Silverstein, 2007) or pursuing diverse types of leisure (Cho et al., 2018) have been associated with older adults' psychosocial wellbeing, and have affected their future outlooks, including mortality rates (Agahi and Parker, 2008). Conversely, some activities have been associated with negative health outcomes. In particular, time spent watching television has been associated with declining cognitive functioning in the older population (Kesse-Guyot et al., 2012).

It seems that different activities may influence different aspects of physical or mental health. For example, productive activities seem to have a better effect on physical functioning, whereas personal hobbies may increase happiness (Menec, 2003). Likewise, the same activities may affect different individuals in different ways. For example, productive activities, such as housework or shopping, were shown to have the greatest impact on reducing the risk of cognitive decline for 
urban women compared to other groups of respondents (Luo et al., 2019). Differences in the benefits derived from the same activities suggest that their effects on individuals might be moderated by certain individual-level characteristics, e.g. activity preferences.

\section{The complex association between daily behaviours, health and subjective wellbeing}

Having rewarding daily activities may promote healthy ageing and help maintain meaningful social relationships (Steptoe and Fancourt, 2019), but at the same time it is conceivable that being healthy or having fulfilling social relations may make daily life better. While numerous observational studies report links between selected activities and wellbeing in later life (e.g. Warr et al., 2004; Litwin and Shiovitz-Ezra, 2006; Nimrod, 2008; Grønning et al., 2018), determining the direction of this association is not straightforward, even in research using longitudinal data (Adams et al., 2011). Overall, most articles assume that an activity promotes wellbeing (Menec, 2003), however, some provide strong evidence for the opposite to be the case, i.e. wellbeing being the causal factor influencing activity participation (Janke et al., 2008).

Intervention studies targeting physical health, psychological functioning, or both, add detail to the picture of complex associations between activities, health and wellbeing. Studies describing interventions targeting both physical and mental health report improved wellbeing post-intervention (Clark et al., 2012). However, this improvement may be due to better physical functioning. Conversely, reports from studies targeting mental health alone do not find lifestyle intervention to be effective in improving or sustaining mental wellbeing (Mountain et al., 2017). As physical and mental health are closely linked, in particular in the population of older adults (Fillenbaum and World Health Organization, 1984), analysing individuals' health profile in more detail might help better understand the association between these variables and daily activities.

Lastly, there are also confounding factors that may affect the relationship between what older adults do and how they feel about their lives or themselves. Additional activity characteristics including, its social context and location, may change the meaning and effect of a given activity, yet those are rarely included in research on wellbeing (Adams et al., 2011). For example, eating typically brings greater joy when it is done with others (Vailas and Nitzke, 1998), and particular eating locations may improve appetite (Stroebele-Benschop et al., 2016). While older adults spend most of their time at home, their overall time spent out of the home has been associated with better physical, cognitive and emotional functioning (Petersen et al., 2015). The broader lifestyle context may also shape the effect of specific activities. For example, the proportion of time individuals spent alone may have an effect on how much enjoyment they derive from their social activities (Klumb, 2004). Likewise, even though being out of the home is seen as generally beneficial for wellbeing, older adults report much higher satisfaction with home-based activities compared to younger individuals (Fortuijn et al., 2006).

This study assumes that it is not a single activity that could make one's life good in later adulthood, but multiple factors operating at the level of daily living, which are reflected in the different things individuals do, as well where and with whom 
they spend their time. These should be analysed together to get a better understanding of the everyday reality of older adults and how it affects them. This study assumes that the main drivers behind individuals' daily behaviours are structural factors, on the one hand, and their health profile, on the other. The assumed influence of structural factors is in line with structural constructivism and the related theoretical approaches, and it is supported by the empirical evidence cited earlier. Health status has been shown to be one of the major predictors of wellbeing in later life, and the degree to which poor health affects daily functioning is seen as the main factor negatively impacting happiness (Angner et al., 2013).

\section{Design and methods}

\section{Data}

The data used in the study came from a sample of 200 non-institutionalised adults aged 65 and above collected at the beginning of 2020 in Poland. Individuals were randomly sampled with quotas for age (using two categories: 65-74, and 75 and over), gender and the size of their place of residence. They were contacted using the Polish telephone directory or approached in public spaces by trained interviewers. A screening questionnaire was used to select eligible respondents. The overall response rate was 27 per cent. The main reason for refusing to participate in the study was due to concerns about sharing one's telephone number for the purpose of data collection; the length of fieldwork was also discouraging. The final sample characteristics are given in Table S1 in the online supplementary material.

Respondents' background information was collected using a standard survey questionnaire. Activity data were collected during five days of fieldwork using the Experience Sampling Method. The Experience Sampling Method records activities in real time, removing memory bias and allowing for a greater accuracy of measures (Csikszentmihalyi and Hunter, 2003). Each respondent received a phone call twice a day, at random times, and was asked about an activity he or she was doing at that time. The calls were distributed across time and days to ensure that all parts of the day were covered for each respondent and that equal representation was achieved for the whole sample. The interviewers asked about their current activity including what it was, where it was happening and who else was present. The dataset is available at Zenodo.org (Jarosz, 2020). All respondents provided written informed consent to participate in the study. The project received ethical clearance from the University of Warsaw Rector's Committee for the Ethics of Research Involving Human Participants.

\section{Measures}

All activities were recorded verbatim and then collapsed into broader categories for the purpose of the study. The information on daily activities was used to construct count variables representing the number of reported activities that fell within each of the categories: (a) necessary, (b) passive, (c) productive, (d) active, (e) social, and (f) leisure pursuits. Necessary activities largely correspond to the conceptualisation of 'necessary time' by Ås (1982), and include sleeping, eating or drinking, and selfcare. Passive activities represent sedentary restful activities requiring little cognitive 
effort, such as passive rest or relaxation, and watching television, with the latter being the most commonly reported primary activity in the dataset. Productive activities represent unpaid work (housework, care work), informal help, running errands (shopping, using services), as well as related travel. Active time denotes any form of physical activity, the most common of which was walking. Social time represents any form of meaningful social interaction, both in person and over the phone or online. Leisure pursuits include leisure activities involving cognitive effort such as hobbies, handicrafts, reading, crosswords and computer use.

The detailed location code was recoded into a count variable representing the number of times a respondent was away from their home. Respondents' own homes were the most frequent location for the reported activities. Two binary variables were constructed to denote whether a respondent reported at least one episode when he or she was accompanied by (a) a family member or (b) by a friend/ acquaintance. The binary variables were used in this case due to the fact that a high share of respondents reported only solitary activities throughout the fieldwork. As the Experience Sampling Method does not allow for the reconstruction of individuals' entire time-use sequences, all of these variables indicate the likelihood of particular events occurring during the day rather than provide exact estimates of their frequency.

Age (continuous) and sex (binary) were used as demographic variables in the models explaining latent class membership. Information on respondents' educational attainment was used in the form of a categorical variable using International Standard Classification of Education-based categories differentiating between individuals with primary and below, secondary and tertiary education. Respondents' occupational class prior to retirement (or during the fieldwork, if the respondent was working at that time) was used as a proxy indicator of their social position with two categories: having held a managerial or professional position (International Standard Classification of Occupations, ISCO codes 1 or 2), or a different occupational position. A binary variable was generated to represent whether a respondent worked for pay at the time of the survey. Living arrangements were represented by an individual's marital status and household size. The primary purpose of the latter variable was to differentiate between individuals who lived alone and those who did not. The variable describing a respondent's area of residence differentiated between 'rural' and 'urban'.

No objective measures of health were available in the dataset, but it included information on self-reported health. The original variable used a five-point Likert scale, varying from very good (5) to very poor (1). In this study, this variable is recorded as a binary variable in which the 'poor' and 'very poor' answers are collapsed into the 'poor health' category, and all other answers into 'average and good health'. In addition to that, a set of binary variables were constructed using the items from a short version of the Nottingham Health Profile (NHP) questionnaire (Kaplan, 2001) which was also collected in the study. The NHP items included statements pertaining to individuals' energy levels: 'Everything is an effort'; and 'I soon run out of energy'. Emotional response was measured using the following items: 'The days seem to drag', 'I have forgotten what it is like to enjoy myself, 'I feel that life is not worth living' and 'I feel I am losing control over my life'. Social isolation was measured using the items: 'I feel lonely', 'I feel there is nobody 
Table 1. Nottingham Health Profile items: distribution of answers, excluding missing

\begin{tabular}{lcccc} 
& Energy levels & Emotional response & Social isolation & Sleep \\
\hline & 43 & Percentages & 77 & 60 \\
\hline (good) & 50 & 40 & 23 & 37 \\
\hline (compromised) & 50 & & 40 & 77 \\
\hline
\end{tabular}

that I am close to' and 'I'm finding it hard to make contact with people'. Finally, sleep disturbances were indicated by one item: 'I sleep badly at night.' Because the study used a short version of the questionnaire, and the NHP does not provide relative-importance weighting for its different dimensions, using these dimensions separately was more informative and easier to interpret in the context of the present research. The four dimensions (energy levels, emotional response, social isolation and sleep disturbances) were thus constructed as binary variables, with 1 corresponding to at least one of the items being answered with 'yes', and 0 meaning the respondent answered 'no' to all items within the given dimension. Frequencies for the four composite variables are given in Table 1.

Lastly, individuals' satisfaction with life in general was used as a proxy for subjective wellbeing. It was measured using the following question: 'How satisfied are you with your life in general?', with answers ranging between not satisfied at all (1) to very satisfied (7).

\section{Analytical approach}

Data on daily activities (activity type, location and co-presence) were used in a latent class analysis (LCA) by fitting the generalised structural equation models in Stata 16 (StataCorp, College Station, TX). A Poisson link was used for count variables (activities, location), and a logit link for binary indicators (co-presence). There were no constraints on estimated parameters. LCA as a method fitted well with the theoretical assumptions of this study. Compared to a cluster analysis, it allows capturing the latent structure behind the data as opposed to merely categorising individuals based on similarities in the observable variables. An additional benefit of LCA is its probabilistic character which allows for selecting the model with optimal fit to the data.

Four models were estimated using different combinations of activities, and specifying two or three classes. In two models the category of necessary activities was excluded due to its highly non-specific character (everyone engages in those activities). The maximum number of classes was set to three due to the low sample size and the fact that individuals' class assignment was used for further analyses. The models were not adjusted for gender, age or health status. These variables were later used to examine class belonging. The model with optimal fit to the data was selected based on the fit statistics, the Bayesian Information Criterion (BIC), the Akaike Information Criterion (AIC), the log likelihood ratio and the interpretative criteria.

Individuals' class membership was then used as an outcome variable in a multinomial logistic regression model. The model used the following explanatory variables: age, gender, self-reported poor health, education, work status, occupational 
position, marital status, household size, area of residence, and detailed measures of individuals' subjective health derived from the NHP - emotional response, energy levels, social isolation and sleep.

Individuals' subjective wellbeing was analysed in relation to their class membership, socio-demographic characteristics and health profile. Nested multivariable linear regression models were used to examine this association. In the first model, wellbeing was regressed on class membership alone. The second model added the rest of the variables. In all of the above models, missing values were retained to preserve the sample size. ${ }^{1}$

\section{Results}

Fit indices for the four models are given in Table S2 in the online supplementary material. Overall, the models which excluded necessary activities, Models 3 and 4, had the best fit to the data. Model 3 assumed the existence of two latent classes while Model 4 specified three classes. In terms of the goodness of fit, the difference between the two models was negligible. The three-class model was chosen based on interpretative grounds. It offered a more nuanced representation of different lifestyles, each of which was distinctive (Figure 1). The probabilities for class membership are given in Table S3 in the online supplementary material, and the proportion of individuals in each class are given in Table 2.

Individuals in Class 1 constituted 30 per cent of the sample. In most cases they were at home during the calls, typically involved in passive activities such as resting and watching television. They also reported engaging in home-based productive activities or leisure pursuits. They were relatively likely to report the presence of a family member. The lifestyle captured by this class was labelled 'passive domiciliary'.

Class 2 was the largest class accounting for 53 per cent of the sample. Individuals' activities were often located outside their homes, and productive activities were reported most often, with passive leisure being the second most frequent type of activity. The third most commonly reported activity was being physically active, which is a distinctive feature of this class compared to other classes. Individuals in Class 2 were likely to be accompanied by a family member but very unlikely to report the presence of a friend. The lifestyle represented by individuals in this class was labelled 'active functional'.

Class 3 was the smallest class, representing 17 per cent of the sample. Individuals in this class were most likely to be involved in productive activities. However, in contrast to Class 2, they also reported a high share of leisure pursuits (reading, games, hobbies) and almost equally as many social activities. Passive activities were almost as numerous as social activities, but with a substantially larger standard error. A distinctive feature of lifestyle represented by this class is a relatively high incidence of reporting the presence of a friend which was more commonly reported than the presence of family members. This lifestyle was labelled 'social recreational'.

Multinomial logistic regression was used to examine the probability of belonging to Class 2 and to Class 3, compared to Class 1. Class 1 was selected as the reference category because the passive domiciliary lifestyle was characterised by the lowest levels of productive, physical and social activity, which are the activities most commonly associated with better health and wellbeing in later life. Furthermore, individuals in this class reported the highest levels of passive rest and were mostly at 

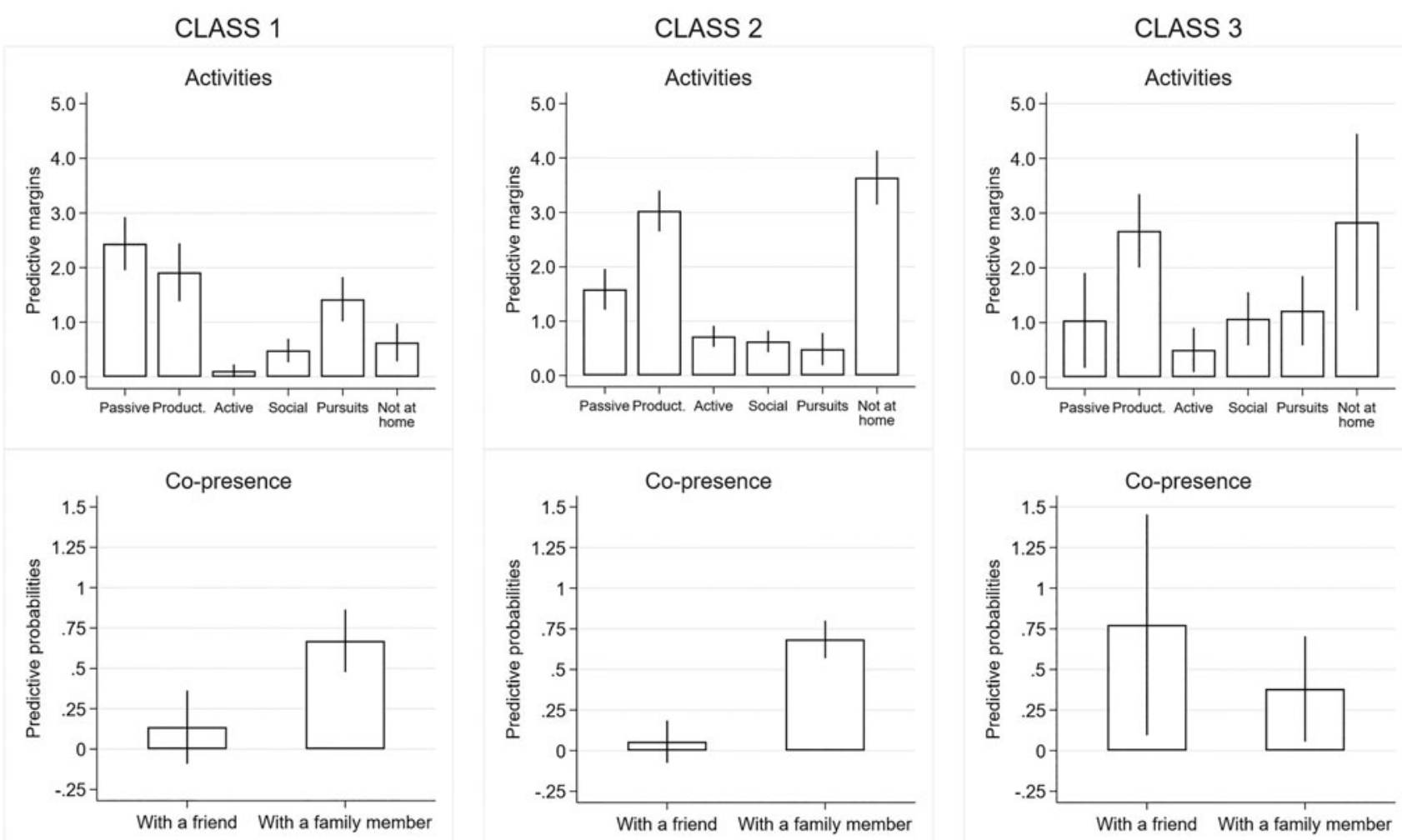

Figure 1. Predictive margins per class.

Notes: Margins at 95 per cent confidence intervals. Product.: productive. 
Table 2. Share of individuals in each class

\begin{tabular}{llcc}
\hline Predicted class & Type of lifestyle (label) & N & $\%$ \\
\hline Class 1 & Passive domiciliary & 61 & 30 \\
\hline Class 2 & Active functional & 105 & 53 \\
\hline Class 3 & Social recreational & 34 & 17 \\
\hline
\end{tabular}

home, which is a potential indicator of some level of functional or other health impairment. The probabilities for class membership are given in Table 3.

Compared to Class 1, individuals in Class 2 were on average younger $(p<0.01)$ and less likely to experience problems with their energy levels $(p<0.05)$. Individuals in Class 3 were also younger than those in Class $1(p<0.05)$, and

Table 3. Class membership - multinomial logistic regression; relative risk ratio for belonging to a given class compared to the reference

\begin{tabular}{|c|c|c|}
\hline & $\begin{array}{c}\text { Class } 2 \text { compared to } \\
\text { Class } 1\end{array}$ & $\begin{array}{c}\text { Class } 3 \text { compared to } \\
\text { Class } 1\end{array}$ \\
\hline & \multicolumn{2}{|c|}{ Relative risk ratios (SE) } \\
\hline Female (Ref. Male) & $0.66(0.27)$ & $2.56(1.57)$ \\
\hline Age & $0.91(0.03)^{\star *}$ & $0.90(0.05)^{*}$ \\
\hline Poor health & $0.52(0.28)$ & $0.56(0.46)$ \\
\hline \multicolumn{3}{|l|}{ Education (Ref. Primary): } \\
\hline Secondary & $2.22(1.12)$ & $1.02(0.74)$ \\
\hline Tertiary & $0.43(0.42)$ & $20.09(27.65)^{\star}$ \\
\hline Working for pay (Ref. Not working) & $0.41(0.26)$ & $0.32(0.31)$ \\
\hline $\begin{array}{l}\text { Managerial/professional occupation } \\
\text { prior to retiring }\end{array}$ & $1.26(1.13)$ & $0.03(0.04)^{*}$ \\
\hline Urban area of residence & $0.42(0.19)$ & $1.60(1.08)$ \\
\hline \multicolumn{3}{|l|}{ Marital status (Ref. Married): } \\
\hline Single & $0.78(0.76)$ & $2.14(2.29)$ \\
\hline Widowed/divorced & $1.45(0.95)$ & $2.31(1.97)$ \\
\hline Single-person household & $3.40(2.30)$ & $3.31(2.82)$ \\
\hline Poor emotional response (Ref. Good) & $1.42(0.74)$ & $0.07(0.06)^{\star \star \star}$ \\
\hline Low energy levels (Ref. Good) & $0.37(0.18)^{\star}$ & $2.86(1.95)$ \\
\hline Social isolation (Ref. Not isolated) & $0.63(0.40)$ & $0.22(0.30)$ \\
\hline Poor sleep (Ref. Good sleep) & $0.93(0.42)$ & $0.95(0.66)$ \\
\hline Intercept & $4,923(12,761)^{\star \star \star}$ & $1,477(5,678)$ \\
\hline $\mathrm{N}$ observations & 200 & 200 \\
\hline
\end{tabular}

Notes: Missing values were retained in the models to preserve sample size, but they are not reported in the table. SE: standard error. Ref.: reference category.

Significance levels : ${ }^{\star} p<0.05,{ }^{\star \star} p<0.01,{ }^{\star \star \star} p<0.001$. 
tended to be better-educated ( $p<0.05)$, but were less likely to have held a managerial or professional position $(p<0.05)$. They were also less likely to report poor emotional response $(p<0.001)$ in their subjective health assessment. Aside from the differences in the detailed dimensions of health as measured by the NHP, the three classes did not differ significantly with regard to the self-reported poor health.

The mean satisfaction with life in the sample was 5.05 (95\% confidence interval $=4.87-5.23$ ), and the distribution of the variable was close to normal. Univariate linear regression models showed significant class differences in the levels of satisfaction with life in general, with Class 1 reporting the lowest wellbeing among all classes (Table 4, Model 1). However, the multivariable model (Table 4, Model 2)

Table 4. Satisfaction with life in general - ordinary least squares (OLS)regression

\begin{tabular}{|c|c|c|}
\hline & Model 1 & Model 2 \\
\hline & \multicolumn{2}{|c|}{ Coefficient (SE) } \\
\hline \multicolumn{3}{|l|}{ Class (Ref. Class 1): } \\
\hline Class 2 & $0.46(0.21)^{\star}$ & $0.07(0.19)$ \\
\hline Class 3 & $0.55(0.27)^{\star}$ & $-0.09(0.26)$ \\
\hline Female gender & - & $-0.07(0.17)$ \\
\hline Age & - & $-0.00(0.01)$ \\
\hline Poor health & - & $-0.79(0.23)^{\star \star \star}$ \\
\hline \multicolumn{3}{|l|}{ Education (Ref. Primary): } \\
\hline Secondary & - & $0.07(0.20)$ \\
\hline Tertiary & - & $-0.06(0.38)$ \\
\hline Working for pay (Ref. Not working) & - & $-0.24(0.26)$ \\
\hline $\begin{array}{l}\text { Managerial/professional occupation prior to } \\
\text { retiring }\end{array}$ & - & $0.31(0.35)$ \\
\hline Urban & - & $0.10(0.18)$ \\
\hline Single-person household & - & $-0.52(0.26)^{\star}$ \\
\hline \multicolumn{3}{|l|}{ Marital status (Ref. Married): } \\
\hline Single & - & $0.35(0.38)$ \\
\hline Divorced/widowed & - & $0.21(0.26)$ \\
\hline Poor emotional response (Ref. Good) & - & $-0.71(0.21)^{\star \star *}$ \\
\hline Low energy levels (Ref. Good) & - & $-0.18(0.21)$ \\
\hline Social isolation (Ref. Not isolated) & - & $-0.42(0.28)$ \\
\hline Poor sleep (Ref. Good sleep) & - & $-0.20(0.19)$ \\
\hline Intercept & $4.72(0.16)^{\star \star \star}$ & $6.10(1.09)^{\star \star \star}$ \\
\hline N observations / Adjusted $R^{2}$ & $200 / 0.02$ & $200 / 0.37$ \\
\hline
\end{tabular}

Notes: Missing values were retained in the models to preserve sample size, but they are not reported in the table. Ref.: reference category.

Significance levels : ${ }^{\star} p<0.05,{ }^{\star \star \star} p<0.001$. 
showed that between-class differences in wellbeing were fully accounted for by individuals' socio-demographic profile and subjective health assessment, i.e. selfreported poor health and emotional response (both significant at $p<0.001$ ). Noteworthy, Model 2 which included socio-demographic and health variables explained a substantial share of the variation in individuals' wellbeing $\left(R^{2}=0.37\right)$ which was a major increase compared to the share of variance explained by Model $1\left(R^{2}=0.02\right)$.

\section{Discussion}

This study used information on everyday activities, their location and social context to look at lifestyle differentiation among older adults. Using LCA, three behavioural classes representing unique lifestyles were identified: passive domiciliary, active functional and social recreational. These lifestyles were associated with distinctive socio-demographic and health profiles. Individuals leading the passive domiciliary lifestyle (Class 1) were on average older, and more likely to report compromised energy levels compared to those in Class 2, or worse emotional response compared to those in Class 3. Individuals leading the active functional lifestyle (Class 2) had good energy levels, were frequently out of the home, often engaged in productive activities and were most likely to be physically active. Individuals leading the social recreational lifestyle (Class 3) were more likely to be tertiary-educated, score high on their emotional response, and socialise or spend time with their friends.

The fact that individuals in Class 2 (active functional) reported better energy levels compared to respondents in Class 1, and individuals in Class 3 (social recreational) had the better emotional response suggests that there was consistency between objectively measured lifestyle characteristics and individuals' subjective assessment of the different aspects of their health. This consistency is worth attention. It supports earlier findings stating that different behaviours or their contextual characteristics may be linked with different aspects of physical or mental health (Menec, 2003).

Earlier studies on the healthy lifestyles of older adults demonstrated that individuals' perceived health status was a predictor of leading a healthy lifestyle, as opposed to demographic variables which predicted a perceived health status but not a health-related lifestyle (Speake et al., 1989). Self-assessment of energy levels or emotional response might also be affected by multiple factors and in a bidirectional manner. For example, greater frequency of social contacts may boost emotional response which in turn could encourage more social interaction. Overall, separating activities from outcomes is challenging as both activity participation and health or wellbeing are likely determined by individuals' characteristics, preferences or habits acquired earlier in life (Adams et al., 2011). However, an important question is whether a change in a person's lifestyle could improve their emotional response. This is particularly relevant because many of the questionnaire items measuring emotional response (such as 'The days seem to drag' or 'I have forgotten what it is like to enjoy myself) de facto represent the subjective assessment of a person's daily activities.

Classes differed with regard to their mean subjective wellbeing in the univariate model, but these differences were fully accounted for by individuals' socio- 
demographic and health profiles. There was also a major difference in the percentage of variance in wellbeing explained by the model including behavioural class alone $\left(R^{2}=0.02\right)$ compared to the model including class, health profile and sociodemographic characteristics $\left(R^{2}=0.37\right)$. There are two possible explanations for that, and they can be seen as complementary. First, these findings may suggest that while respondents' lifestyles may be associated with wellbeing, this association is largely due to factors that determine the daily behaviours of older adults, such as their health, including its different psychosocial dimensions. This is in line with earlier evidence suggesting that health limitations may impact wellbeing through the way they affect the daily functioning of older adults (Angner et al., 2013). An alternative explanation would be one in line with earlier studies on the Valuation of Life (e.g. Jopp et al., 2008) which argue that different activities may be selected to boost meaning and maximise wellbeing by different individuals. Older adults' diverse lifestyles would thus reflect their particular lifestage and habitus, which seems likely taking into account the significant age and education effects on class belonging. Lifestyles are also affected by health, but not in a deterministic manner. That means older adults can still do what they consider meaningful or enjoyable but they may be limited in their choices. Nonetheless, some of the lifestyles identified in this article are potentially more likely to correspond to the concept of 'engaged lifestyle' (Rowe and Kahn, 1997), and their link with individuals' health status is eminent.

\section{Limitations}

The cross-sectional design of this study does not allow a causal relationship between the variables to be established. This is a major weakness since it is not known to what extent particular dimensions of individuals' health affect their daily activities, and to what extent it is lifestyle and daily experiences that shapes individuals' health perception.

The sample size was small, which resulted in the fact that many differences between the classes were statistically non-significant due to large standard errors. Potentially, more classes could be specified with a larger sample, and differences between them could be analysed in greater detail. The measures of NHP did not use the full long version of the questionnaire which could give a more detailed picture of the associations between particular dimensions of health and individuals' lifestyles. Furthermore, the detailed NHP measures were dichotomised for the models as opposed to being used as continuous scales, which made the estimates less precise. However, this was necessary due to the limitations resulting from the small sample size.

Information about respondents' functional or cognitive impairment or additional indicators of subjective wellbeing would permit more nuanced analyses. The present study also did not account for the subjective characteristics of analysed activities, such as how much respondents actually enjoyed what they did; that has been done elsewhere (Jarosz, 2021). Earlier research on older adults (Scott et al., 2015) demonstrated that the same activity may carry different meaning for different individuals, and various aspects of a particular activity may be emphasised. Possibly, the meaning attached to an activity may also moderate its effect on an individual's wellbeing. 


\section{Conclusions}

This study captures various dimensions of lifestyle differentiation at an older age. It identifies three distinct behavioural classes and finds an association between individuals' lifestyles, their socio-demographic characteristics and health profile. Individuals who engage in multiple productive activities over the day and are frequently out of home report good energy levels; socially engaged individuals display good emotional response.

The association between individuals' lifestyle and their subjective wellbeing was significant in the univariate models. However, in the multivariable models the class effects were accounted for by other characteristics, primarily related to health. Overall, while daily lifestyles are differentiated by individuals' age, education and health profile, they are not the decisive factor in shaping older adults' subjective wellbeing.

Supplementary material. The supplementary material for this article can be found at https://doi.org/10. $1017 /$ S0144686X21001586

Acknowledgements. I am deeply grateful to all respondents, many of whom were frail and at an advanced age, without whose dedication and commitment this study would not have been possible.

Financial support. This work was supported by the European Union's Horizon 2020 Research and Innovation Programme (grant agreement number 840113).

Conflict of interest. The author declares no conflicts of interest.

Ethical standards. The study received ethics clearance from the University of Warsaw Rector's Committee for the Ethics of Research Involving Human Participants (approval number 44/2019). Written informed consent was obtained from all respondents.

\section{Note}

1 There were only single cases of missing values for behavioural and socio-demographic variables. A higher number of missing values was recorded for the items in the NHP, with the overall highest number of missing values being recorded for social isolation (ten). There were eight missing values for emotional response, seven for energy levels and six for sleep.

\section{References}

Adams KB (2004) Changing investment in activities and interests in elders' lives: theory and measurement. International Journal of Aging and Human Development 58, 87-108.

Adams KB, Leibbrandt S and Moon H (2011) A critical review of the literature on social and leisure activity and wellbeing in later life. Ageing \& Society 31, 683-712.

Agahi N and Parker MG (2008) Leisure activities and mortality. Journal of Aging and Health 20, 855-871. Angner E, Ghandhi J, Williams Purvis K, Amante D and Allison J (2013) Daily functioning, health status, and happiness in older adults. Journal of Happiness Studies 14, 1563-1574.

Ås D (1982) Designs for large scale time use studies of the 24-hour day. In Straikov Z (ed). It's About Time. Sofia: Institute of Sociology at the Bulgarian Academy of Sciences, Bulgarian Sociological Association, pp. $17-53$.

Ayalon L (2009) Family and family-like interactions in households with round-the-clock paid foreign carers in Israel. Ageing \& Society 29, 671-686.

Baltes PB and Baltes MM (1990) Psychological perspectives on successful aging: the model of selective optimization with compensation. In Baltes PB and Baltes MM (eds), Successful Aging: Perspectives from the Behavioral Sciences. Cambridge: Cambridge University Press, pp. 1-34.

Bourdieu P (1984) Distinction: A Social Critique of the Judgement of Taste. London: Routledge. 
Chen B, Covinsky KE, Cenzer IS, Adler N and Williams BA (2012) Subjective social status and functional decline in older adults. Journal of General Internal Medicine 27, 693-699.

Chen J-H, Waite LJ and Lauderdale DS (2015) Marriage, relationship quality, and sleep among U.S. older adults. Journal of Health and Social Behavior 56, 356-377.

Cho D, Post J and Kim SK (2018) Comparison of passive and active leisure activities and life satisfaction with aging. Geriatrics \& Gerontology International 18, 380-386.

Clark F, Jackson J, Carlson M, Chou C-P, Cherry BJ, Jordan-Marsh M, Knight BG, Mandel D, Blanchard J, Granger DA, Wilcox RR, Lai MY, White B, Hay J, Lam C, Marterella A and Azen SP (2012) Effectiveness of a lifestyle intervention in promoting the well-being of independently living older people: results of the Well Elderly 2 Randomised Controlled Trial. Journal of Epidemiology and Community Health 66, 782-790.

Cockerham W (1999) Health and Social Change in Russia and Eastern Europe. New York: Routledge.

Csikszentmihalyi M and Hunter J (2003) Happiness in everyday life: the uses of experience sampling. Journal of Happiness Studies 4, 185-199.

Drew LM and Silverstein M (2007) Grandparents' psychological well-being after loss of contact with their grandchildren. Journal of Family Psychology 21, 372-379.

Espinel PT, Chau JY, van der Ploeg HP and Merom D (2015) Older adults' time in sedentary, light and moderate intensity activities and correlates: application of Australian Time Use Survey. Journal of Science and Medicine in Sport 18, 161-166.

Fillenbaum GG and World Health Organization (1984) The Wellbeing of the Elderly: Approaches to Multidimensional Assessment. Geneva: World Health Organization.

Fortuijn JD, van der Meer M, Burholt V, Ferring D, Quattrini S, Hallberg IR, Weber G and Wenger GC (2006) The activity patterns of older adults: a cross-sectional study in six European countries. Population, Space and Place 12, 353-369.

Gauthier AH and Smeeding TM (2003) Time use at older ages. Research on Aging 25, 247-274.

Grønning K, Espnes GA, Nguyen C, Rodrigues AMF, Gregorio MJ, Sousa R, Canhão H and André B (2018) Psychological distress in elderly people is associated with diet, wellbeing, health status, social support and physical functioning - a HUNT3 study. BMC Geriatrics 18, 205.

Havighurst RJ (1961) Successful aging. The Gerontologist 1, 8-13.

Janke MC, Nimrod G and Kleiber DA (2008) Reduction in leisure activity and well-being during the transition to widowhood. Journal of Women \& Aging 20, 83-98.

Jarosz E (2020) DEXSAGE Daily Experiences of Successful Ageing. Version v1 (Dataset). Available at https://zenodo.org/record/4017865\#.YWhj3xpByM8.

Jarosz E (2021) What makes life enjoyable at an older age? Experiential wellbeing, daily activities, and satisfaction with life in general. Aging \& Mental Health Available online doi. 10.1080/13607863.2021. 1916879.

Jopp D, Rott C and Oswald F (2008) Valuation of life in old and very old age: the role of sociodemographic, social, and health resources for positive adaptation. The Gerontologist 48, 646-658.

Kahana E and Kahana B (1996) Conceptual and empirical advances in understanding aging well through proactive adaptation. In Bengtson VL (ed.), Adulthood and Aging: Research on Continuities and Discontinuities. New York, NY: Springer, pp. 18-40.

Kaplan RM (2001) Health outcomes, assessment of. In Smelser Neil and Baltes Paul (eds). International Encyclopedia of the Social \& Behavioral Sciences. Amsterdam: Elsevier, pp. 6581-6586.

Kesse-Guyot E, Charreire H, Andreeva VA, Touvier M, Hercberg S, Galan P and Oppert J-M (2012) Cross-sectional and longitudinal associations of different sedentary behaviors with cognitive performance in older adults. PLOS ONE 7, e47831.

Klumb PL (2004) Benefits from productive and consumptive activities: results from the Berlin Aging Study. Social Indicators Research 67, 107-127.

Lawton MP, Moss M, Hoffman C, Grant R, Have TT and Kleban MH (1999) Health, valuation of life, and the wish to live. The Gerontologist 39, 406-416.

Lawton MP, Moss MS, Winter L and Hoffman C (2002) Motivation in later life: personal projects and well-being. Psychology and Aging 17, 539-547.

Lemon BW, Bengtson VL and Peterson JA (1972) An exploration of the activity theory of aging: activity types and life satisfaction among in-movers to a retirement community. Journal of Gerontology 27, 511-523. 
Li H (2006) Rural older adults' access barriers to in-home and community-based services. Social Work Research 30, 109-118.

Litwin $\mathbf{H}$ and Shiovitz-Ezra S (2006) The association between activity and wellbeing in later life: what really matters? Ageing \& Society 26, 225-242.

Luo Y, Pan X and Zhang Z (2019) Productive activities and cognitive decline among older adults in China: evidence from the China Health and Retirement Longitudinal Study. Social Science \& Medicine 229, 96-105.

Menec VH (2003) The relation between everyday activities and successful aging: a 6-year longitudinal study. Journals of Gerontology: Psychological Sciences and Social Sciences 58B, S74-S82.

Moss MS, Hoffman CJ, Mossey J and Rovine M (2007) Changes over 4 years in health, quality of life, mental health, and valuation of life. Journal of Aging and Health 19, 1025-1044.

Mountain G, Windle G, Hind D, Walters S, Keertharuth A, Chatters R, Sprange K, Craig C, Cook S, Lee E, Chater T, Woods R, Newbould L, Powell L, Shortland K and Roberts J (2017) A preventative lifestyle intervention for older adults (lifestyle matters): a randomised controlled trial. Age and Ageing 46, 627-634.

Mullen SP, Olson EA, Phillips SM, Szabo AN, Wójcicki TR, Mailey EL, Gothe NP, Fanning JT, Kramer AF and McAuley E (2011) Measuring enjoyment of physical activity in older adults: invariance of the physical activity enjoyment scale (paces) across groups and time. International Journal of Behavioral Nutrition and Physical Activity 8, 103.

Nimrod G (2008) In support of innovation theory: innovation in activity patterns and life satisfaction among recently retired individuals. Ageing \& Society 28, 831-846.

Petersen J, Austin D, Mattek N and Kaye J (2015) Time out-of-home and cognitive, physical, and emotional wellbeing of older adults: a longitudinal mixed effects model. PLOS ONE 10, e0139643.

Raphael D, Brown I, Renwick R and Rootman I (1997) Quality of life: what are the implications for health promotion? American Journal of Health Behavior 21, 118-128.

Rowe J and Kahn R (1987) Human aging: usual and successful. Science 237, 143-149.

Rowe JW and Kahn RL (1997) Successful aging. The Gerontologist 37, 433-440.

Scott TL, Masser BM and Pachana NA (2015) Exploring the health and wellbeing benefits of gardening for older adults. Ageing \& Society 35, 2176-2200.

Sobel ME (1981) Lifestyle and Social Structure: Concepts, Definitions, Analyses. New York, NY: Academic Press.

Speake DL, Cowart ME and Pellet K (1989) Health perceptions and lifestyles of the elderly. Research in Nursing \& Health 12, 93-100.

Steptoe A and Fancourt D (2019) Leading a meaningful life at older ages and its relationship with social engagement, prosperity, health, biology, and time use. Proceedings of the National Academy of Sciences 116, 1207-1212.

Stroebele-Benschop N, Depa J and de Castro JM (2016) Environmental strategies to promote food intake in older adults: a narrative review. Journal of Nutrition in Gerontology and Geriatrics 35, 95-112.

Talmage CA, Coon DW, Dugger BN, Knopf RC, O'Connor KA and Schofield SA (2020) Social leisure activity, physical activity, and valuation of life: findings from a longevity study. Activities, Adaptation \& Aging 44, 61-84.

Vailas LI and Nitzke SA (1998) Food enjoyment scale for older adults. Journal of Nutrition for the Elderly $17,59-64$.

Warr P, Butcher V and Robertson I (2004) Activity and psychological well-being in older people. Aging \& Mental Health 8, 172-183.

Yaffe K, Barnes D, Nevitt M, Lui L-Y and Covinsky K (2001) A prospective study of physical activity and cognitive decline in elderly women. Archives of Internal Medicine 161, 1703-1708.

Zhu X, Qiu C, Zeng Y and Li J (2017) Leisure activities, education, and cognitive impairment in Chinese older adults: a population-based longitudinal study. International Psychogeriatrics 29, 727-739.

Cite this article: Jarosz E (2021). Lifestyle differentiation among older adults: exploring the links between individuals' behaviours, socio-demographic characteristics, health and wellbeing in later life. Ageing \& Society 1-16. https://doi.org/10.1017/S0144686X21001586 